\title{
Gastroprotective Effects of PMK-S005 against Ethanol-Induced Acute Gastric Damage in Rats
}

Yoon Jeong Choi*, Nayoung Kim**, ${ }^{\dagger}$ Ju Yup Lee*, Ryoung Hee Nam*, Ji Hyung Seo*, Seonmin Lee*, Hee Jin Kim*, Yoon Jin Choi*, Hye Seung Lee ${ }^{\ddagger}$, and Dong Ho Lee ${ }^{*}{ }^{\dagger}$

*Department of Internal Medicine, Seoul National University Bundang Hospital, Seongnam, ${ }^{\dagger}$ Department of Internal Medicine and Liver Research Institute, Seoul National University College of Medicine, Seoul, and ${ }^{\ddagger}$ Department of Pathology, Seoul National University Bundang Hospital, Seongnam, Korea

See editorial on page 321.

Background/Aims: This study aimed to examine the gastroprotective effects of PMK-S005, which is a synthetic S-allyl-Lcysteine (SAC; a sulfur-containing amino acid), against acute ethanol-induced gastric damage in rats. Methods: SpragueDawley rats were divided into six groups, including a nonethanol group, groups treated with absolute ethanol 1 hour after pretreatment with various doses of PMK-S005 (1, 5, and 10 $\mathrm{mg} / \mathrm{kg}$ ) or rebamipide (50 mg/kg), and an absolute ethanolonly group. Ethanol-induced gross ulcer and mucus levels were measured. Myeloperoxidase, tumor necrosis factor $\alpha$, interleukin 1 $\beta, \mathrm{PGE}_{2}, \mathrm{LTB}_{4}, \mathrm{CPLA}_{2}, \mathrm{COX}-1$, and COX-2 levels were estimated by enzyme-linked immunosorbent assay or Western blot analysis. Furthermore, the protein expression levels of antioxidant enzymes, including heme oxygenase-1 (HO-1), NAD(P)H:quinine oxidoreductase 1 (NQO-1), GCLC, and GCLM, were assessed. Results: PMK-S005 significantly attenuated the ethanol-induced gastric damage; it reduced mucosal inflammatory cytokine production and increased mucus levels. The expression levels of $\mathrm{CPLA}_{2}, \mathrm{COX}-1$, and COX-2 were decreased by PMK-S005. PMK-S005 did not affect $\mathrm{PGE}_{2}$ synthesis, but $\mathrm{LTB}_{4}$ production was significantly suppressed. In addition, long-term administration of PMKS005 significantly increased the expression of HO-1, NQO-1, GCLC, and GCLM. Conclusions: These results strongly suggest that PMK-SO05 prevents gastric mucosal damage and that these gastroprotective activities are due to anti-inflammatory effects and enhancement of the gastric defense system, including antioxidant enzymes. (Gut Liver 2016;10:348355)
Key Words: S-allyl-L-cysteine; Gastroprotection; Anti-inflammation; Antioxidants; Ethanol

\section{INTRODUCTION}

The gastric mucosal layers are constantly exposed to potentially noxious substances, hydrochloric acid, and proteolytic enzyme, but the stomach usually maintains its structural and functional integrity because of tight intercellular junctions, secretion of bicarbonate, and growth factors for epithelial renewal and circulation. ${ }^{1}$ However, a variety of agents can cause gastric injury if they overwhelm mucosal defense mechanism. Gastric ulcers are predominantly caused by infection with Helicobacter pylori, nonsteroidal anti-inflammatory drugs (NSAIDs), alcohol consumption, and cigarette smoking. ${ }^{2-4}$ Oral consumption of excessive alcohol results in necrotic lesions in the gastric mucosa through producing acute hemorrhagic lesions, mucosal edema, epithelial exfoliation, inflammatory cell infiltration, which in turn reduces defensive factors such as bicarbonate secretion and mucus production. ${ }^{5}$ Therefore, ethanol-induced gastric damage is a useful model for evaluation of ethanol-induced pathologic changes in gastric mucosa. ${ }^{6}$

In addition, reactive oxygen species (ROS) play a important role in the development of pathogenesis related to acute gastric damage induced by stress, ethanol, and NSAIDs. ${ }^{7,8}$ Ethanol reduces levels of nonprotein sulfhydryl groups such as gluthathion (GSH), thereby increasing ROS which demonstrate ulcerogenic activity., ${ }^{9,10}$ To cope with noxious insults, cellular defense system is induced to detoxify and eliminate those harmful chemicals and their metabolites. Expression of cytoprotective enzymes such as heme oxygenase-1 (HO-1), NAD(P)H:quinine

Correspondence to: Nayoung Kim

Department of Internal Medicine, Seoul National University Bundang Hospital, 82 Gumi-ro 173beon-gil, Bundang-gu, Seongnam 463-707, Korea

Tel: +82-31-787-7008, Fax: +82-31-787-4051, E-mail: nayoungkim49@empas.com

Received on December 24, 2014. Revised on March 20, 2015. Accepted on March 27, 2015. Published online September 9 , 2015 pISSN 1976-2283 eISSN 2005-1212 http://dx.doi.org/10.5009/gnl14509

(a) This is an Open Access article distributed under the terms of the Creative Commons Attribution Non-Commercial License (http://creativecommons.org/licenses/by-nc/4.0) which permits unrestricted non-commercial use, distribution, and reproduction in any medium, provided the original work is properly cited. 
oxidoreductase 1 (NQ0-1), catalytic (GCLC) and modulator (GCLM) subunits of rate-limiting enzyme in glutathione biosynthesis ( $\gamma$-glutamyl-cystein ligase), is believed to be implicated in protecting cells against oxidative stress and other toxic insults.

$S$-allyl-L-cysteine (SAC), the most abundant organosulfur molecule in aged garlic extract, has long been used as a common dietary supplement and traditional medicine. PMK-S005 is a synthetic SAC which is sulfur containing water soluble amino acid. SAC has been reported to have multiple biological effects such as antioxidant, anti-inflammation, anticancer and antihepatotoxic activities. ${ }^{11-13}$ Recently, we reported that the gastroprotective effects of PMK-S005 against NSAIDs-induced acute gastric damage rat model. ${ }^{14}$ Now we evaluate the effect of PMK-S005 on the gastroprotective effects in an ethanol-induced acute gastric damage rat model. In addition, the molecular mechanisms underlying its gastroprotective effects in the aspect of proinflammatory cytokines, mucin synthesis, arachidonic acid pathway, as well as antioxidant enzyme were comprehensively examined.

\section{MATERIALS AND METHODS}

\section{Animals}

Seven-week-old male Sprague-Dawley rats (Orient Co., Ltd., Seoul, Korea) were housed in a cage maintained at $23^{\circ} \mathrm{C}, 12 / 12-$ hour light/dark cycle under specific pathogen-free conditions. After 1 week of adaptation, 8-week-old rats weighing 250 to $300 \mathrm{~g}$ were used for the experiments. All experimental procedures were approved by the Institutional Animal Care and Use Committee (IACUC) of Seoul National University Bundang Hospital (IACUC number: BA1304-127-033-01). The rats were starved but allowed water for 12 hours prior to the experiments.

\section{Ethanol-induced gastric mucosal injury model}

Either PMK-SO05 (1, 5, $10 \mathrm{mg} / \mathrm{kg}$ ) or rebamipide (50 mg/kg) was administrated orally to rats, which had been fasted for 12 hours, 1 hour prior to the induction of gastric damage. Acute gastric lesions were induced by intragastric administration of $100 \%$ ethanol ( $5 \mathrm{~mL} / \mathrm{kg}$ ). After 1 hour (ethanol), the animals were sacrificed, and the gastric lesions were scored.

\section{Gross ulcer index and histological index}

After sacrificing the animals, the isolated stomachs were cut open along the greater curvature and washed in ice-cold saline. To investigate the degree of gross mucosal damage, the mucosal sides of the stomachs were photographed using a digital camera, and part of the mucosa was immediately fixed with 10\% formalin solution. The gross damage of the gastric mucosa was assessed by three gastroenterologists, who were blinded to the treatments, using a gross ulcer index. ${ }^{15}$ In addition, the mucosal damage was graded by assigning an index of histological injury. ${ }^{16}$

\section{Measurement of adherent mucus and hexosamine}

The concentration of adherent gastric mucosal mucus was expressed as the concentration of Alcian blue adhering to the gastric mucosal surface ( $\mu \mathrm{g} / \mathrm{g}$ tissue) ${ }^{17}$ The excised stomach was extensively washed with ice-cold saline, and gastric mucosa was scraped with a slide glass and frozen at $-70^{\circ} \mathrm{C}$ until use. Finally, hexosamine appropriately obtained from hydrolyzed mucin was assayed using the method described by Neuhaus et al. ${ }^{18}$

\section{Measurement of mucosal myeloperoxidase, tumor necrosis factor $\alpha$, and interleukin $1 \beta$}

An enzyme-linked immunosorbent assay (ELISA) was performed to measure the level of the cytokine expression using the appropriate kits from HyCult Biotechnology (Uden, The Netherlands) and R\&D Systems (Minneapolis, MN, USA) by following the manufacturer's instructions. All assays were performed in triplicate, and data are shown as mean \pm standard errors (SEM).

\section{Measurement of mucosal PGE 2 and $\mathrm{LTB}_{4}$}

$\mathrm{PGE}_{2}$ and $\mathrm{LTB}_{4}$ concentration in gastric mucosa was measured with a commercial kit (Cayman Chemical, Ann Arbor, MI, USA) Briefly, the scraped mucosa was weighed and placed in a tube containing 100\% ethanol plus $0.1 \mathrm{M}$ indomethacin. The samples were then minced with scissors, homogenized and centrifuged at $13,000 \mathrm{rpm}$ for 10 minutes at $4^{\circ} \mathrm{C}$. The supernatant of each sample was used for the determination of $\mathrm{PGE}_{2}$ and $\mathrm{LTB}_{4}$.

\section{Western blotting for cPLA $_{2}$, COX-1, COX-2, HO-1, NQO-1, GCLC, and GCLM}

Equal amounts of protein from gastric mucosal lysates were subjected to SDS-PAGE analysis, and immunoblotting using the appropriate antibodies was performed as we previously described. ${ }^{15}$ The incubation condition for each antibody is as follows: anti-cPLA ${ }_{2}$ antibody (1:1,000; Santa Cruz Biotechnology, Santa Cruz, CA, USA), anti-COX-1 antibody (1:1,000; Santa Cruz Biotechnology), anti-COX-2 antibody (1:1,000, Cayman Chemical), anti-HO-1 antibody (1:1,000; Abcam Inc., Cambridge, UK), anti-NQO-1 antibody (1:1,000; Abcam Inc.), antiGCLC (1:1,000; Abcam Inc.), or anti-GCLM (1:1,000; Abcam Inc.).

\section{Statistical analysis}

Data are expressed as the mean \pm SEM. Statistical analyses were conducted with GraphPad Prism (GraphPad Software, La Jolla, CA, USA) and SPSS software version 12.0 (SPSS Inc., Chicago, IL, USA). Statistical significance was determined using the Mann-Whitney $U$ test and $\mathrm{p}<0.05$ was considered to be statistically significant. 

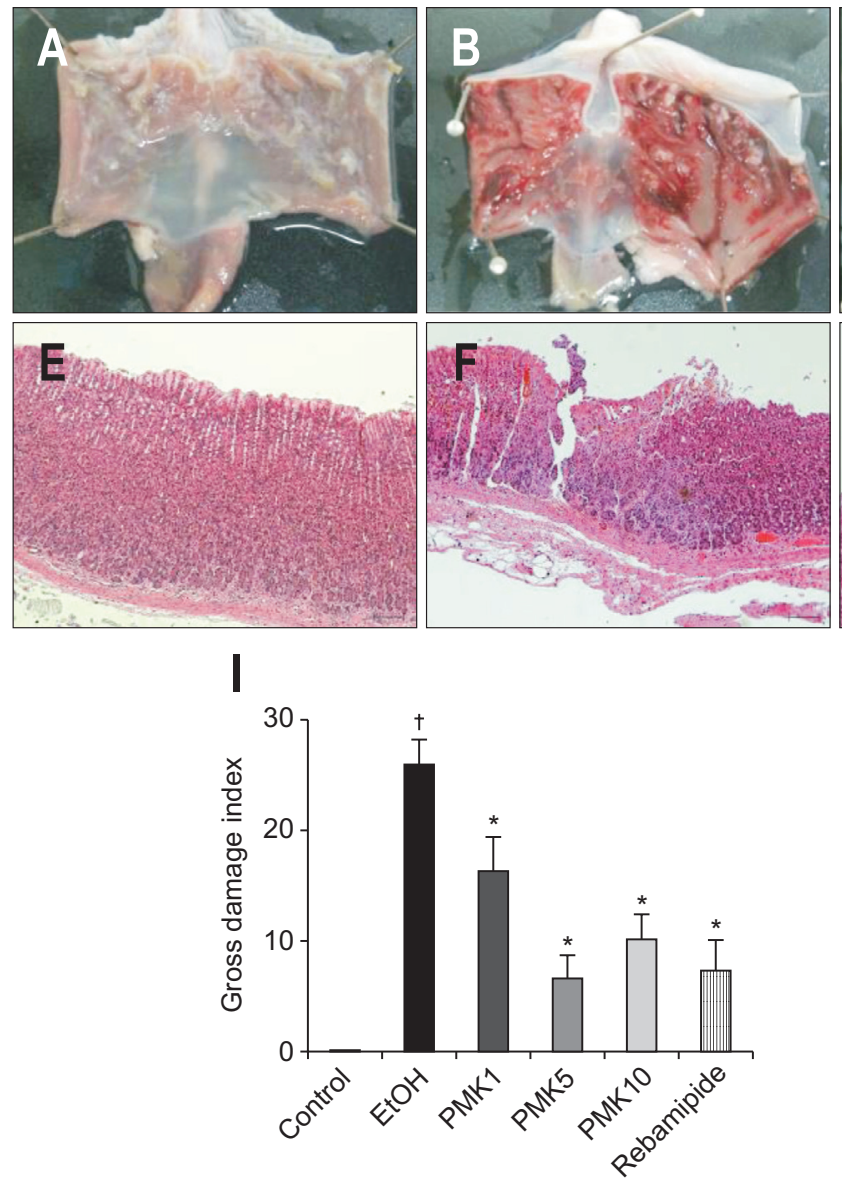
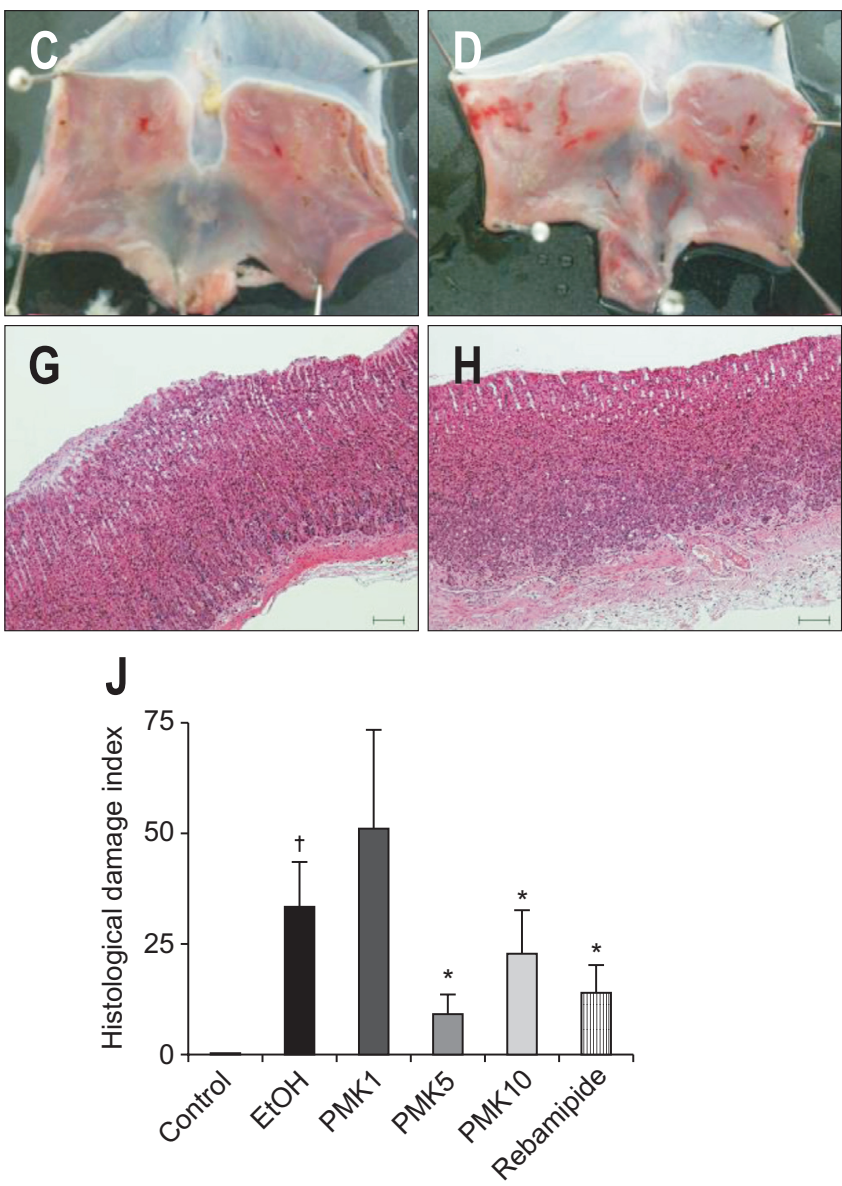

Fig. 1. Gross (A-D) and histological (E-H) findings after ethanol-induced gastric damage of the rat stomach. Rat stomachs (A, E) were damaged by ethanol ( $5 \mathrm{~mL} / \mathrm{kg}$ ) (B, F), and a significant portion of the damage was prevented by pretreatment with $5 \mathrm{mg} / \mathrm{kg}$ PMK-S005 (C, G) or $50 \mathrm{mg} / \mathrm{kg}$ rebamipide (D, H) 1 hour before intragastric ethanol administration. Macroscopic damage indices and histological damage indices were quantified (I, J). $\mathrm{Bar}, 200 \mu \mathrm{m}$. The results are expressed as the mean \pm SEM from five to 10 animals per group. ${ }^{*} \mathrm{p}<0.05$ compared with the ethanol group; ${ }^{\dagger} \mathrm{p}<0.05$ compared with the control group.

\section{RESULTS}

\section{Effect of PMK-S005 on acute gastric lesions induced by ethanol}

Absolute ethanol induced severe gastric damage (Fig. 1B and F) and this gastric damage was attenuated by the PMK-S005 or rebamipide pretreatment (Fig. 1A-H). The gross ulcer indices reduced from 25.8 to $16.3,6.57$, and 10.0 after pretreatment with PMK-S005 at a dose of 1,5 , and $10 \mathrm{mg} / \mathrm{kg}$, respectively ( $\mathrm{p}=0.029$, $\mathrm{p}<0.001$, and $\mathrm{p}<0.001$, respectively) (Fig. 1I). In addition, pretreatment with $5 \mathrm{mg} / \mathrm{kg}$ of PMK-S005 significantly reduced the histological indices compared with those in the ethanol group (9.3, $\mathrm{p}=0.109$ ) (Fig. 1J). The inhibition rate of $5 \mathrm{mg} / \mathrm{kg}$ of PMKS005 for gastric lesions was higher than that of $50 \mathrm{mg} / \mathrm{kg}$ of rebamipide.

\section{Effect of PMK-S005 on hexosamine and adherent mucus in ethanol-induced gastric damage}

The hemorrhagic erosion caused by ethanol administration is related to the destruction of the mucus barrier. Thus, we measured the gastric mucosal hexosamine concentration, an index for gastric mucus synthesis, and adherent mucus, an indicator of gastric mucus secretion. Intragastric administration of ethanol significantly decreased gastric mucosal hexosamine and adherent mucus concentration compared to the control rat (Fig. 2A and B). This ethanol-induced reduction of hexosamine and adherent mucus concentrations was markedly prevented by pretreatment with $5 \mathrm{mg} / \mathrm{kg}$ of PMK-S005 (Fig. 2A and B), which was similar to the histological result.

\section{Effect of PMK-S005 on myeloperoxidase, tumor necrosis factor $\alpha$, and interleukin $1 \beta$ level in ethanol-induced gas- tric damage}

Increased mucosal levels of myeloperoxidase (MP0) after ethanol administration (Fig. 2C) were suppressed by pretreatment with $5 \mathrm{mg} / \mathrm{kg}$ or $10 \mathrm{mg} / \mathrm{kg}$ of PMK-S005. Rebamipide pretreatment also suppressed the ethanol-stimulated MPO level to $173.2 \mathrm{ng} / \mathrm{mg}$ protein. Increased mucosal tumor necrosis factor a (TNF- $\alpha$ ) concentration (Fig. 2D) and interleukin $1 \beta$ (IL$1 \beta$ ) concentration (Fig. 2E) after ethanol administration were 


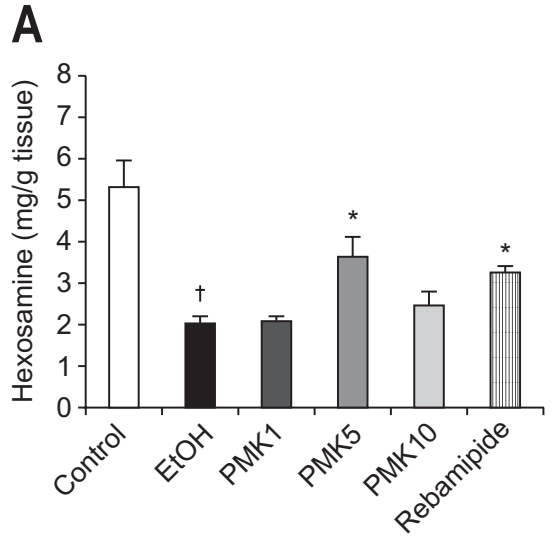

C

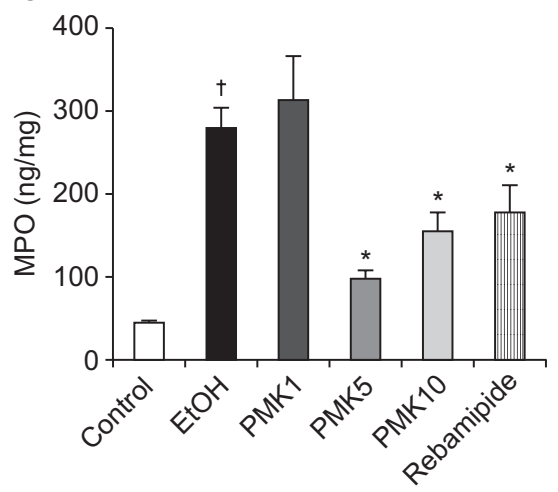

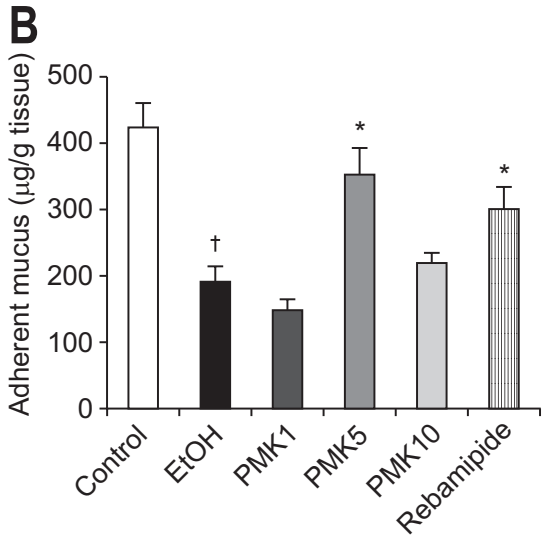

E
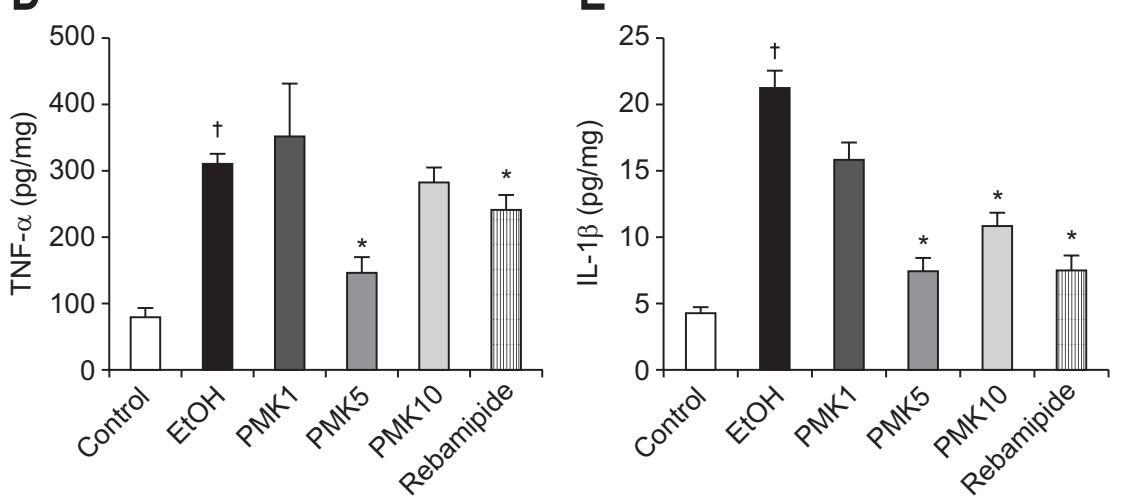

Fig. 2. Gastric mucus levels and anti-inflammatory activities of PMK-S005 in ethanol-induced gastric damage. Hexosamine concentrations (A) and adherent mucus (B) were significantly decreased after the intragastric administration of ethanol, and these reductions were markedly relieved by pretreatment with $5 \mathrm{mg} / \mathrm{kg}$ of PMK-S005. The ethanol-administered rats showed marked increases in myeloperoxidase (MP0) (C), tumor necrosis factor $\alpha$ (TNF- $\alpha$ ) (D), and interleukin $1 \beta$ (IL-1 $\beta$ ) levels (E). These increases were significantly inhibited by pretreatment with $5 \mathrm{mg} / \mathrm{kg}$ PMKS005. The results are expressed as the mean \pm SEM from five to 10 animals per group. ${ }^{*} p<0.05$ compared with the ethanol group; ${ }^{\dagger} p<0.05$ compared with the control group.

significantly suppressed by pretreatment with $5 \mathrm{mg} / \mathrm{kg}$ of PMKS005 or rebamipide (Fig. 2D and E).

\section{Western blotting of $\mathrm{CPLA}_{2}$, COX-1, and COX-2}

The significantly increased expression of $\mathrm{CPLA}_{2}(0.82)$ in the rat stomachs 1 hour after administration of ethanol (Fig. 3A) was reduced by PMK-S005 pretreatment to 0.27 ( $5 \mathrm{mg} / \mathrm{kg}, \mathrm{p}=0.002$ ) or $0.41(10 \mathrm{mg} / \mathrm{kg}, \mathrm{p}=0.014)$. Similar to $\mathrm{CPLA}_{2}$, the increased COX-1 (Fig. 3B) and COX-2 expression (Fig. 3C) was also significantly suppressed by pretreatment with $5 \mathrm{mg} / \mathrm{kg}$ or $10 \mathrm{mg} / \mathrm{kg}$ of PMK-S005. Rebamipide pretreatment also significantly decreased the ethanol-induced cPLA 2 , COX-1, and COX-2 expressions.

\section{Effect of PMK-S005 on $\mathrm{PGE}_{2}$ and $\mathrm{LTB}_{4}$ level in ethanol- induced gastric damage}

Since our Western blot results indicated that pretreatment

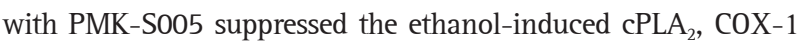
and COX-2 expression, we examined further downstream factors of inflammatory responses. The mucosal level of $\mathrm{PGE}_{2}$ was not significantly changed after ethanol administration, and oral administration of PMK-S005 did not affect the mucosal $\mathrm{PGE}_{2}$ level (Fig. 3D). In contrast to $\mathrm{PGE}_{2}$, the increased mucosal level of $\mathrm{LTB}_{4}$ after ethanol administration was decreased by pretreatment with PMK-S005 at a concentration of 5 and $10 \mathrm{mg} / \mathrm{kg}$ $(\mathrm{p}<0.001)$ and rebamipide (Fig. 3E).

\section{Effect of PMK-S005 on gastric H0-1, NQO-1, GCLC, and GCLM expression}

In the next, we determined whether PMK-S005 could potentiate the antioxidative capacity of gastric mucosa. Rats fed with PMK-S005 (5 or $10 \mathrm{mg} / \mathrm{kg}$ ) for 14 days showed no histological change in gastric tissue (data not shown). The protein levels of HO-1, NQO-1, GCLC, and GCLM were significantly increased in the rat stomachs in PMK-S005-treated rats compared with the control rats (Fig. 4).

\section{DISCUSSION}

A variety of plant-derived flavonoids and their derivates have been demonstrated to have the gastroprotective effects. ${ }^{19-21}$ Garlic (Allium sativum) has long been used as a common dietary 
A
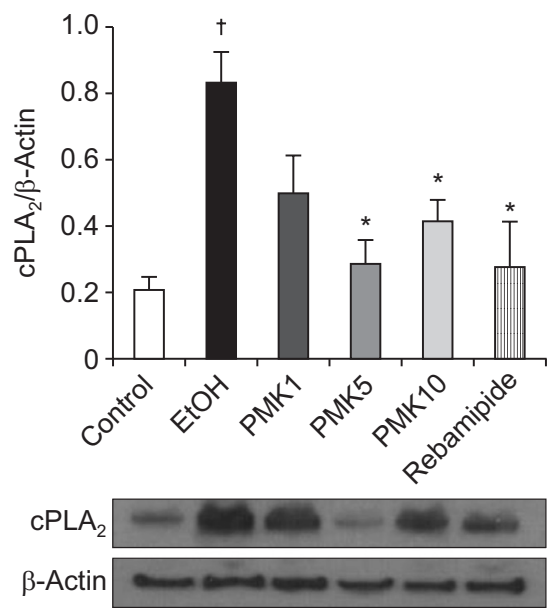

B
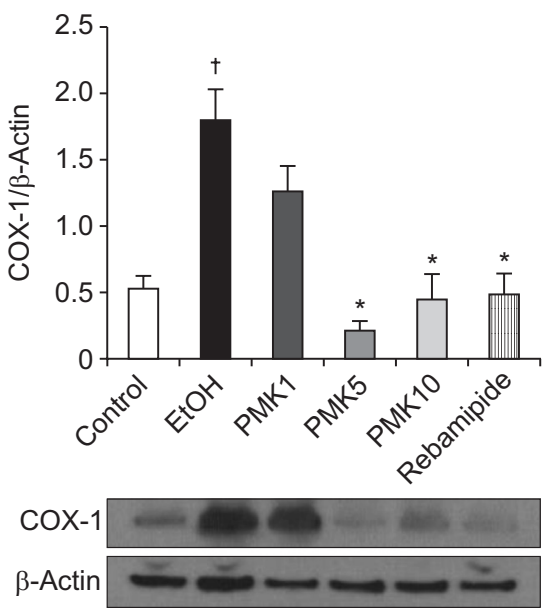

C

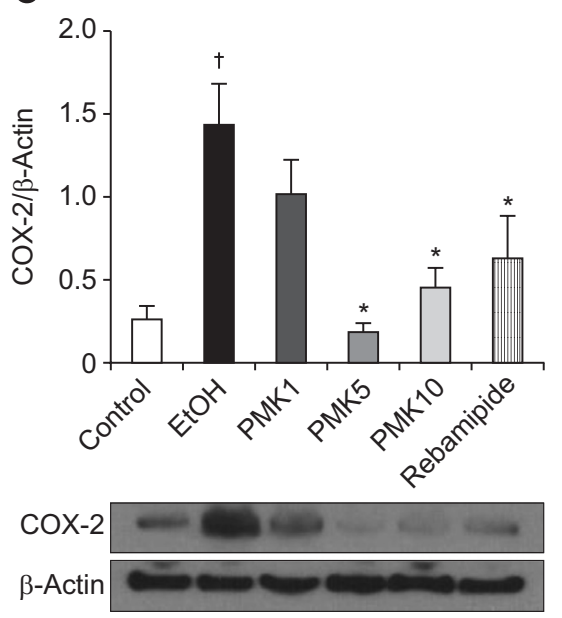

D

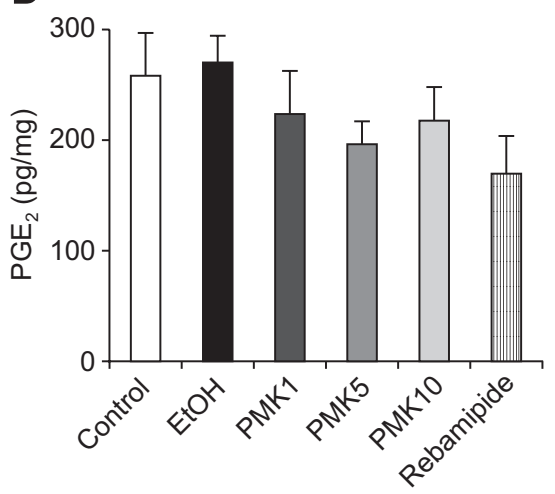

E

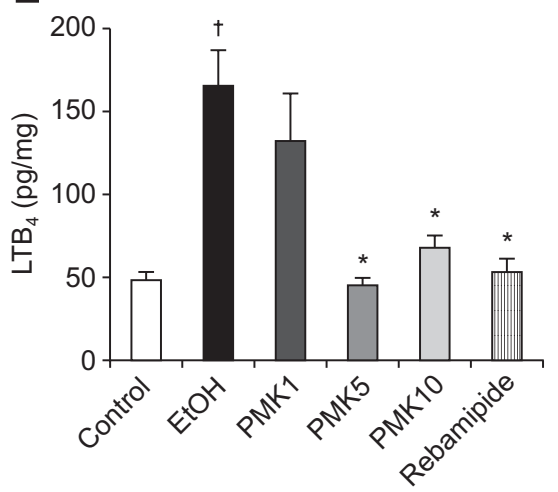

Fig. 3. Western blot analysis of $\mathrm{CPLA}_{2}$ (A), COX-1 (B), and COX-2 (C) expression in the rat gastric mucosa damaged by ethanol; the mucosal concentrations of $\mathrm{PGE}_{2}(\mathrm{D})$ and $\mathrm{LTB}_{4}(\mathrm{E})$ are also shown. The ethanol-administered rats showed marked increases in cPLA $(\mathrm{A}), \mathrm{COX}-1$ (B), and COX-2 (C) expression in the gastric mucosa (a representative blot is shown). These increases were significantly suppressed by pretreatment with PMK-S005 $\left(5 \mathrm{mg} / \mathrm{kg}\right.$ ) or rebamipide (50 mg/kg), which were given 1 hour before ethanol administration. The mucosal concentrations of PGE (D) and LTB $_{4}(\mathrm{E})$ were determined 1 hour after ethanol administration using an enzyme-linked immunosorbent assay. The results are expressed as the mean \pm SEM from five to 10 animals per group. ${ }^{*} \mathrm{p}<0.05$ compared with the ethanol group; ${ }^{\dagger} \mathrm{p}<0.05$ compared with the control group.

supplement and traditional medicine. Garlic constituents has been reported to have multiple biological function including antioxidant activity, antithrombotic activity, lowering blood lipid, cardiovascular effects, improvement of immune function, and anticancer effect. ${ }^{22}$ Garlic contains several organosulfur compounds and water soluble SAC is the most abundant organosulfur compound with anti-inflammatory and antioxidant properties. ${ }^{23}$ However, the mechanism underlying the protective effects of SAC against gastric damage is not fully understood.

Although we recently reported that the gastroprotective effect of PMK-S005 in NSAIDs-induced gastric damage model, ${ }^{14}$ we investigated its action on ethanol-induced ulcer model in order to provide more evidence concerning the gastroprotective effect of PMK-S005. In the present study, we report two major findings. First, the PMK-S005 pretreatment exert gastroprotective action against ethanol-induced gastric damage via attenuating the lesions produced by ethanol, inducing gastric mucus level, decreasing production of proinflammatory cytokines, and reducing the expression of $\mathrm{CPLA}_{2}, \mathrm{COX}-1, \mathrm{COX}-2$, and $\mathrm{LTB}_{4}$ after ethanol administration. Although this effect did not show to be dose dependent and the gastroprotective activity was somehow decreased at a concentration of $10 \mathrm{mg} / \mathrm{kg}$, all effects of PMKS005 were consistently maximized at the concentration of 5 $\mathrm{mg} / \mathrm{kg}$, which was more potent to rebamipide (50 mg/kg). This result suggests that less than $10 \mathrm{mg} / \mathrm{kg}$ PMK-S005 treatment afforded significantly preventive effect against ethanol-induced gastric damages. To analyze the protective effect of PMK more clearly, rebamipide was adopted for comparison that has been developed in Japan as a promising gastroprotective drug and is widely prescribed for treating peptic ulcers. ${ }^{24}$ Dosage of rebamipide used here was selected considering previous animal study. ${ }^{25,26}$ Second, long-term administration of PMK-S005 (5 or $10 \mathrm{mg} / \mathrm{kg}$ ) induces the expression of antioxidant enzyme such as HO-1, NQO-1, GCLC, and GCLM in gastric mucosa. 

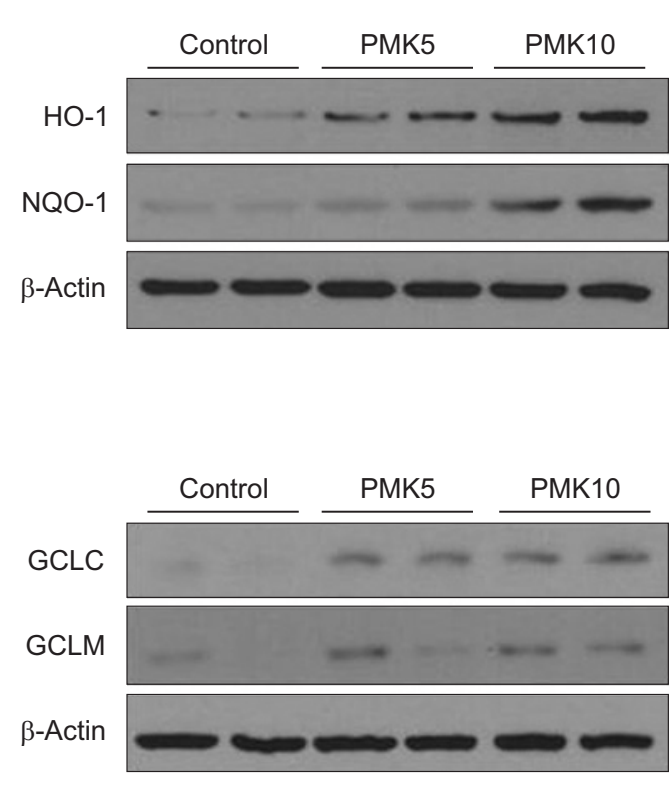
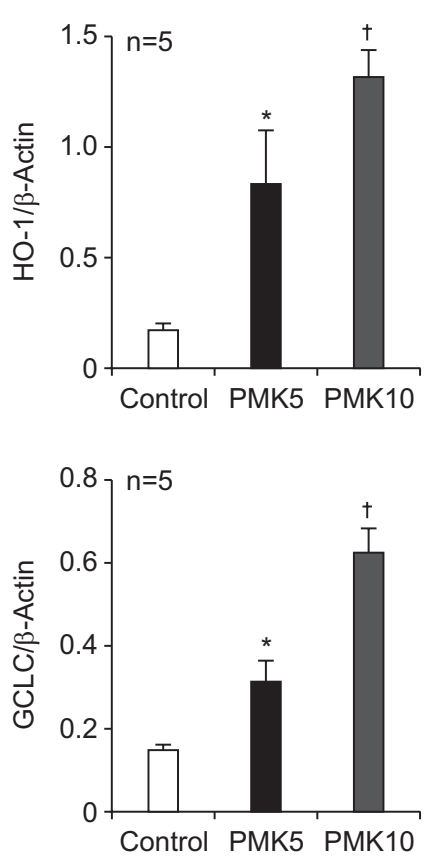
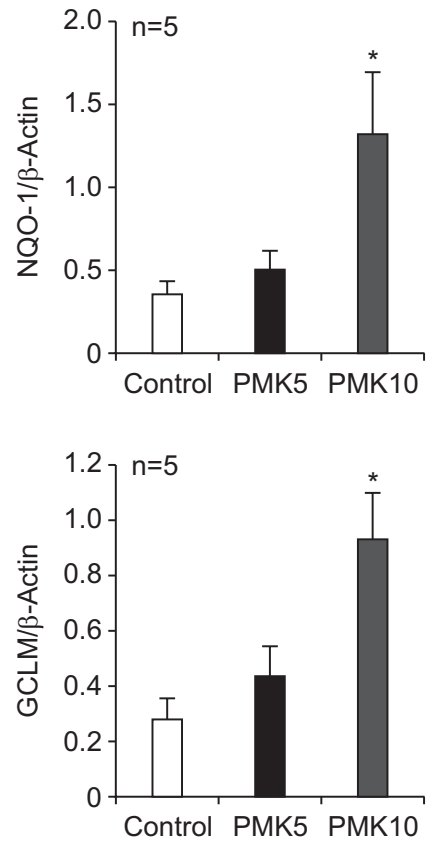

Fig. 4. PMK-S005 induces the expression of antioxidant enzymes in the rat stomach. Western blot analysis of heme oxygenase-1 (HO-1), NAD(P) H:quinine oxidoreductase 1 (NQO-1), GCLC, and GCLM levels in the rat gastric mucosa of control and PMK-S005 (5 or $10 \mathrm{mg} / \mathrm{kg})-$ treated rats. HO-1, NQO-1, GCLC, and GCLM levels were significantly increased in PMK-S005 (5 or $10 \mathrm{mg} / \mathrm{kg}$ )-treated rats. The results are expressed as the mean \pm SEM from five animals per group. ${ }^{*} \mathrm{p}<0.05$ and ${ }^{\dagger} \mathrm{p}<0.01$ compared with the control group.

Ethanol-induced gastric damage has been widely used for the evaluation regarding the gastroprotective activities of drugs. Acute ethanol challenge induces oxidative stress and lipid peroxidation, and destroys the mucosal barrier, leading to the depletion of gastric wall mucus. ${ }^{5,27,28}$ Our results showed that pretreatment with PMK-S005 significantly enhanced gastric adherent mucus and hexosamine levels, suggesting that the protection of gastric mucus by PMK-S005 might be one the mechanisms responsible for its protective effects. This result was also confirmed in NSAIDs-induced ulcer model. Consequently, we speculate that gastroprotective effect of PMK-S005 may be due to partly to its beneficial action on the defensive factors such as mucus barrier and partly to its inhibitory effect on inflammatory components of ethanol- and NSAIDs-induced gastric damages. Although further studies are required to elucidate precise mechanism involved in gastric mucus protection, several factors such as mucin (MUC) gene expression, NO, and gastric sensory afferents are considered to be might contribute to the protection of gastric mucus.

There are two pathways to metabolize the arachidonic acid, which is released from membrane phospholipids in the inflammation process. One is prostaglandins synthesis through COX and the other one is leukotriene synthesis through 5-LOX. In this study, we found that increase of cPLA $\mathrm{P}_{2}$ expression after ethanol administration was attenuated by PMK-S005 pretreatment. Furthermore, the decrease of $\mathrm{LTB}_{4}$ production, by-product of 5-LOX and CPLA ${ }_{2}$, in the gastric mucosa represents the reduced downstream of cPLA ${ }_{2}$ expression. In addition, PMK-S005 pre- treatment inhibited ethanol-induced MPO and proinflammatory cytokines including TNF- $\alpha$ and IL-1 $\beta$ levels in the gastric mucosa. These results suggest that PMK-S005 has anti-inflammatory activity by inhibiting cPLA 2 signaling. However, PMK-S005 seems to have nothing to do with $\mathrm{PGE}_{2}$ in ethanol-induced gastric damage because $\mathrm{PGE}_{2}$ production was not changed in the gastric mucosa despite overexpression of COXs (Fig. 3D). These results are similar to previous studies. ${ }^{29,30}$ It might be explained that in the presence of oxidative damage caused by ethanol, the activity of COXs enzyme is inhibited or prostaglandins could be converted into products of oxidation such as 8 -iso- $\mathrm{PGF}_{2} \mathrm{\alpha}$ which results in no significant changes in their metabolite $\mathrm{PGE}_{2}$ production. ${ }^{31,32}$

Several reports indicate that SAC is a potent antioxidant agent and prevent to the oxidative damage observed in chronic degenerative diseases. ${ }^{33}$ In this study, we also evaluated the gastroprotective effects of PMK-S005 in the aspect of antioxidant as well as anti-inflammatory action. We previously found that PMK-S005 treatment induced the expression of antioxidant enzyme such as HO-1 and superoxide dismutase-1 in vitro system (unpublished data). As demonstrated in Fig. 4, long-term administration of PMK-S005 also induced the expression of antioxidant enzyme such as HO-1 and NQO-1 in gastric mucosa in vivo. These findings suggest that oral administration of PMK-S005 could maintain the gastric epithelial barrier through antioxidative activity thus conferring protection against gastric ulcer. Recently, many studies have demonstrated that HO-1 is implicated in cytoprotective mechanism via antioxidant, anti- 
inflammatory, antiproliferative, and antiapoptotic properties. ${ }^{34-38}$ We also noticed that treatment of mice with PMK-S005 (10 mg/ $\mathrm{kg} /$ day) for 14 days did not produced any adverse gastric reactions. However, the absorption and metabolism of PMK-S005 remain largely unknown and need further investigation in the context of the potential application of this PMK-S005 in the management of human gastric ulcer.

In conclusion, the anti-inflammatory, cytoprotective, and antioxidative activities of PMK-S005 impose significant protective efficacy against ethanol-induced gastric mucosal injury. Actually, phase II clinical study of PMK-S005 to confirm the effective dose in human gastric ulcer and for a new drug of gastric distress is conducting. In the safety evaluation, no clinically meaningful events were observed for vital sign, physical examination. Therefore, clinical evidence is required to show the real clinical utility of PMK-S005 for the prevention and treatment of human gastric injury.

\section{CONFLICTS OF INTEREST}

No potential conflict of interest relevant to this article was reported.

\section{ACKNOWLEDGEMENTS}

This work was supported by Chungcheong leading industry promotion project of the Korean Ministry of Knowledge Economy.

\section{REFERENCES}

1. Flemström G, Isenberg JI. Gastroduodenal mucosal alkaline secretion and mucosal protection. News Physiol Sci 2001;16:23-28.

2. Laine L, Takeuchi K, Tarnawski A. Gastric mucosal defense and cytoprotection: bench to bedside. Gastroenterology 2008;135:4160.

3. Srivastava A, Lauwers GY. Pathology of non-infective gastritis. Histopathology 2007;50:15-29.

4. Franke A, Teyssen S, Singer MV. Alcohol-related diseases of the esophagus and stomach. Dig Dis 2005;23:204-213.

5. Guslandi M. Effects of ethanol on the gastric mucosa. Dig Dis 1987;5:21-32.

6. Goso Y, Ueno M, Hotta K, Ishihara K. Protective effects of the whisky congeners on ethanol-induced gastric mucosal damage. Alcohol Clin Exp Res 2007;31:390-394.

7. Banerjee RK. Nonsteroidal anti-inflammatory drugs inhibit gastric peroxidase activity. Biochim Biophys Acta 1990;1034:275-280.

8. Das D, Bandyopadhyay D, Bhattacharjee M, Banerjee RK. Hydroxyl radical is the major causative factor in stress-induced gastric ulceration. Free Radic Biol Med 1997;23:8-18.

9. Mizui T, Sato H, Hirose F, Doteuchi M. Effect of antiperoxidative drugs on gastric damage induced by ethanol in rats. Life Sci 1987;
41:755-763.

10. Ajaikumar KB, Asheef M, Babu BH, Padikkala J. The inhibition of gastric mucosal injury by Punicagranatum L. (pomegranate) methanolic extract. J Ethnopharmacol 2005;96:171-176.

11. Ray B, Chauhan NB, Lahiri DK. Oxidative insults to neurons and synapse are prevented by aged garlic extract and S-allyl-L-cysteine treatment in the neuronal culture and APP-Tg mouse model. $\mathrm{J}$ Neurochem 2011;117:388-402.

12. Mong MC, Yin MC. Nuclear factor kappaB-dependent anti-inflammatory effects of s-allyl cysteine and s-propyl cysteine in kidney of diabetic mice. J Agric Food Chem 2012;60:3158-3165.

13. Thomson M, Ali M. Garlic [Allium sativum]: a review of its potential use as an anti-cancer agent. Curr Cancer Drug Targets 2003;3 67-81.

14. Choi YJ, Kim N, Lee JY, et al. Protective effects of garlic extract, PMK-S005, against nonsteroidal anti-inflammatory drugs-induced acute gastric damage in rats. Dig Dis Sci 2014;59:2927-2934.

15. Nam SY, Kim N, Lee CS, et al. Gastric mucosal protection via enhancement of MUC5AC and MUC6 by geranylgeranylacetone. Dig Dis Sci 2005;50:2110-2120.

16. Lacy ER, Ito S. Microscopic analysis of ethanol damage to rat gastric mucosa after treatment with a prostaglandin. Gastroenterology 1982;83:619-625.

17. Kitagawa H, Takeda F, Kohei H. A simple method for estimation of gastric mucus and effects of antiulcerogenic agents on the decrease in mucus during water-immersion stress in rats. Arzneimittelforschung 1986;36:1240-1244.

18. Nishida K, Ohta Y, Ishiguro I. Teprenone, an anti-ulcer agent, increases gastric mucosal mucus level via nitric oxide in rats. Jpn J Pharmacol 1998;78:519-522.

19. Martín MJ, Marhuenda E, Pérez-Guerrero C, Franco JM. Antiulcer effect of naringin on gastric lesions induced by ethanol in rats Pharmacology 1994;49:144-150.

20. Mota KS, Dias GE, Pinto ME, et al. Flavonoids with gastroprotective activity. Molecules 2009;14:979-1012.

21. Lim JH, Kim JH, Kim N, et al. Gastroprotective effect of Cochinchina momordica seed extract in nonsteroidal anti-inflammatory drug-induced acute gastric damage in a rat model. Gut Liver 2014;8:49-57.

22. Borek C. Antioxidant health effects of aged garlic extract. J Nutr 2001;131(3S):1010S-1015S.

23. Maldonado PD, Barrera D, Rivero I, et al. Antioxidant S-allylcysteine prevents gentamicin-induced oxidative stress and renal damage. Free Radic Biol Med 2003;35:317-324.

24. Arakawa T, Higuchi K, Fujiwara Y, et al. 15th anniversary of rebamipide: looking ahead to the new mechanisms and new applications. Dig Dis Sci 2005;50 Suppl 1:S3-S11.

25. Choi SM, Shin JH, Kang KK, Ahn BO, Yoo M. Gastroprotective effects of DA-6034, a new flavonoid derivative, in various gastric mucosal damage models. Dig Dis Sci 2007;52:3075-3080.

26. Choi HS, Lim JY, Chun HJ, et al. The effect of polaprezinc on gastric mucosal protection in rats with ethanol-induced gastric muco- 
sal damage: comparison study with rebamipide. Life Sci 2013;93: 69-77.

27. Bagchi D, Carryl OR, Tran MX, et al. Stress, diet and alcoholinduced oxidative gastrointestinal mucosal injury in rats and protection by bismuth subsalicylate. J Appl Toxicol 1998;18:3-13.

28. Robert A, Nezamis JE, Lancaster C, Hanchar AJ. Cytoprotection by prostaglandins in rats. Prevention of gastric necrosis produced by alcohol, $\mathrm{HCl}, \mathrm{NaOH}$, hypertonic $\mathrm{NaCl}$, and thermal injury. Gastroenterology 1979;77:433-443.

29. Asako H, Kubes P, Wallace J, Gaginella T, Wolf RE, Granger DN. Indomethacin-induced leukocyte adhesion in mesenteric venules: role of lipoxygenase products. Am J Physiol 1992;262(5 Pt 1): G903-G908.

30. Gambero A, Maróstica M, Becker TL, Pedrazzoli J Jr. Effect of different cyclooxygenase inhibitors on gastric adaptive cytoprotection induced by 20\% ethanol. Dig Dis Sci 2007;52:425-433.

31. Natale G, Lazzeri G, Lubrano V, et al. Mechanisms of gastroprotection by lansoprazole pretreatment against experimentally induced injury in rats: role of mucosal oxidative damage and sulfhydryl compounds. Toxicol Appl Pharmacol 2004;195:62-72.

32. Fujimoto Y, Uno E, Sakuma S. Effects of reactive oxygen and nitrogen species on cyclooxygenase-1 and -2 activities. Prostaglan- dins Leukot Essent Fatty Acids 2004;71:335-340

33. Colín-González AL, Santana RA, Silva-Islas CA, Chánez-Cárdenas ME, Santamaria A, Maldonado PD. The antioxidant mechanisms underlying the aged garlic extract-and S-allylcysteine-induced protection. Oxid Med Cell Longev 2012;2012:907162.

34. Takahashi T, Morita K, Akagi R, Sassa S. Heme oxygenase-1: a novel therapeutic target in oxidative tissue injuries. Curr Med Chem 2004;11:1545-1561.

35. Zhou H, Lu F, Latham C, Zander DS, Visner GA. Heme oxygenase-1 expression in human lungs with cystic fibrosis and cytoprotective effects against Pseudomonas aeruginosa in vitro. Am J Respir Crit Care Med 2004;170:633-640.

36. Kapturczak MH, Wasserfall C, Brusko T, et al. Heme oxygenase-1 modulates early inflammatory responses: evidence from the heme oxygenase-1-deficient mouse. Am J Pathol 2004;165:1045-1053.

37. Deng YM, Wu BJ, Witting PK, Stocker R. Probucol protects against smooth muscle cell proliferation by upregulating heme oxygenase-1. Circulation 2004;110:1855-1860.

38. Pae HO, Choi BM, Oh GS, et al. Roles of heme oxygenase-1 in the antiproliferative and antiapoptotic effects of nitric oxide on Jurkat T cells. Mol Pharmacol 2004;66:122-128. 